Original Article $\quad$ www.pjkd.com.pk

\title{
Clinical Presentation and Outcomes of Covid-19 in Maintenance Hemodialysis Patients in Pakistan: A Single-Center Study
}

\author{
Sonia Yaqub, Amna Hamid, Zarghoona Naeem, Safia Awan \\ Section of Nephrology, \\ Department of Medicine, \\ Aga Khan University, Karachi, Pakistan.
}

\begin{abstract}
OBJECTIVE: The objective of the present study was to review clinical presentation and short-term outcomes of COVID-19 in patients on maintenance hemodialysis (MHD).

STUDY DESIGN: Retrospective observational study

PLACE AND DURATION OF STUDY: The study was conducted at three coordinated outpatient hemodialysis units at Aga Khan University Hospital from 1st May 2020 to 31st July, 2020.

METHODOLOGY: Data on baseline characteristics, clinical features, laboratory and radiographic findings, treatment and short come outcomes were analyzed for 15 patients on MHD diagnosed with COVID-19 during the study period. All characteristics were also compared between surviving and deceased subjects.

RESULTS: Out of 108 MHD patients, 17 were diagnosed with COVID-19 through polymerase chain reaction (PCR) and 15 of them were included in the study. Mean age of subjects was 62.8 years; $53.3 \%$ were females. Comorbidities were hypertension (100\%), diabetes mellitus (86.7\%) and cardiovascular disease (33.3\%). Median HD vintage was 15 months (IQR 8-14). Forty percent of the patients were asymptomatic. Frequent symptoms observed were shortness of breath (40\%), cough (26.7\%) and fever (20\%). Lymphopenia was not seen in our study and $40 \%$ had no radiographic finding. Acute respiratory distress syndrome, myocardial injury, hepatic dysfunction and thrombotic event were observed complications. Compared to survivors, deceased patients were significantly older $(\mathrm{p}=0.01)$ and had higher inflammatory markers. Forty percent were hospitalized and $83.3 \%$ required ventilation (invasive or noninvasive). By the end of follow up; $40 \%$ patients died.

CONCLUSION: Patients on MHD are susceptible to severe COVID-19 and higher mortality. Asymptomatic presentation demands high clinical suspicion for prompt diagnosis. Following trend of inflammatory markers may help clinician in identification of severe cases and appropriate management.
\end{abstract}

Key Words: COVID-19, hemodialysis, presentation, outcome, mortality

\section{Corresponding Author:}

Dr. Sonia Yaqub

Assistant professor

Director Nephrology Residency Program

Department of Medicine

Aga Khan University

Karachi

Email: Sonia.yaqub@aku.edu

\section{Introduction:}

Coronavirus Disease 2019 (COVID-19) is a global pandemic caused by novel severe acute respiratory syndrome coronavirus 2 (SARS-CoV2). It has a wide clinical spectrum with multi-organ involvement leading to severe morbidity and mortality. ${ }^{1}$ Epidemiological surveys have shown old age and comorbid conditions such as hypertension (HTN), diabetes mellitus (DM), cardiovascular disease (CVD) and chronic kidney disease (CKD) increase susceptibility to severe disease. ${ }^{2,3}$ However, the impact of COVID-19 in population on hemodialysis (HD) is still unclear. Nephrologists anticipate patients on maintenance hemodialysis (MHD) could be vulnerable to severe disease and poor outcome. Dialysis patients are commonly affected with above-mentioned comorbidities ${ }^{4}$ and are also prone to infection 
due to uremia-induced suppressed immunity. ${ }^{5,6}$ Moreover, even though ambulatory restrictions and social distancing are important to prevent COVID-19, patients with end stage renal disease (ESRD) are bound to visit hemodialysis units twice or thrice weekly for life sustaining treatment and therefore have increased risk of exposure to contagion.

Few studies including case series and single-center experiences from United States, China and Europe have reported varying prevalence, clinical presentations and case fatalities. Prevalence ranging 2-28\% with regional variations and a high mortality rate $14-51 \%$ has been observed compared to general population. ${ }^{7-15}$ However, pattern and outcome of COVID-19 in Pakistan and other developing countries is not known where limitation of resources may also impact the disease course and outcome. Thereby, we aim to survey presentation of covid-19 and its short-term outcome in MHD patients in an urban outpatient hemodialysis center in Pakistan which may improve clinician awareness and help them in effective management.

\section{Patients and Methods}

This was a retrospective observational study of COVID-19 in patients on hemodialysis at three coordinated outpatient hemodialysis units (HDU) in Aga Khan University Hospital, Karachi. A total of 108 patients receive maintenance hemodialysis (MHD) twice or thrice weekly in our hemodialysis facility. Since the first reported case of COVID-19 in our HDU in May 2020, strict implementation of Centre for Disease Control (CDC) recommended measures to mitigate risk of COVID-19 outbreak ${ }^{16}$ were ensured in all three units. These measures included mandatory wearing of face mask by all within premises as well as upgrade personal protective equipment (PPE) for staff, physical distancing in waiting areas and dialysis units, and a two-step screening process for signs and symptoms of COVID.

Initial screening was done on telephone a day prior to scheduled session followed by another assessment by HDU staff before entering the hemodialysis units. Nasopharyngeal swab samples for nucleic acid testing of SARS-CoV2 were collected from all suspected cases. The nucleic acid testing was conducted in clinical laboratory of Aga khan University Hospital by using fluorescent real time reverse transcriptase polymerase chain reaction (RT-PCR) kits for SARS-CoV-2. All positive cases were isolated and dialyzed separately in a room dedicated for COVID-19 patients. Additional work up was done at the discretion of attending physician that included complete blood count, C-reactive protein (CRP), ferritin, lactate dehydrogenase (LDH) and procalcitonin (PCT) levels as markers of inflammation, D-dimer marker for vascular injury, Troponin I (Trop I) for myocardial injury, aspartate and alanine aminotransferases (AST and ALT) as indicator of hepatic dysfunction.

During the study period, from $1^{\text {st }}$ May to $31^{\text {st }}$ July, 2020, 38 patients were suspected for COVID-19 whereas 17 of them tested positive for SARS-CoV2 RT-PCR. One patient was excluded due to history of dialysis at another facility prior to testing positive. Another patient with acute on chronic kidney disease was also excluded (Figure 1). Fifteen patients were included in final analysis, 10 of whom were diagnosed until early June followed by a decline in positive cases and only one case was identified in July, Figure 2.

Data including demographic information, medical history, exposure history, symptoms, signs, laboratory and radiological findings, treatment, complications and outcomes were obtained from the electronic medical record system and file record of Aga Khan University Hospital. An independent trained physician collected the data which was double checked by another nephrologist in research team. Need for hospitalization and length of stay, need for mechanical ventilation, death or discharge from hospital where taken as short come outcomes in our study. The clinical outcomes were monitored up to 
$31^{\text {st }}$ July, 2020, the final date of follow-up. This study was approved by Institutional review board of Aga Khan University. Informed consent was waived, and strictly anonymized data was analyzed.

Figure:1 flow diagram showing identification of patients with COVID-19 amongst 108 maintenance hemodialysis patients.

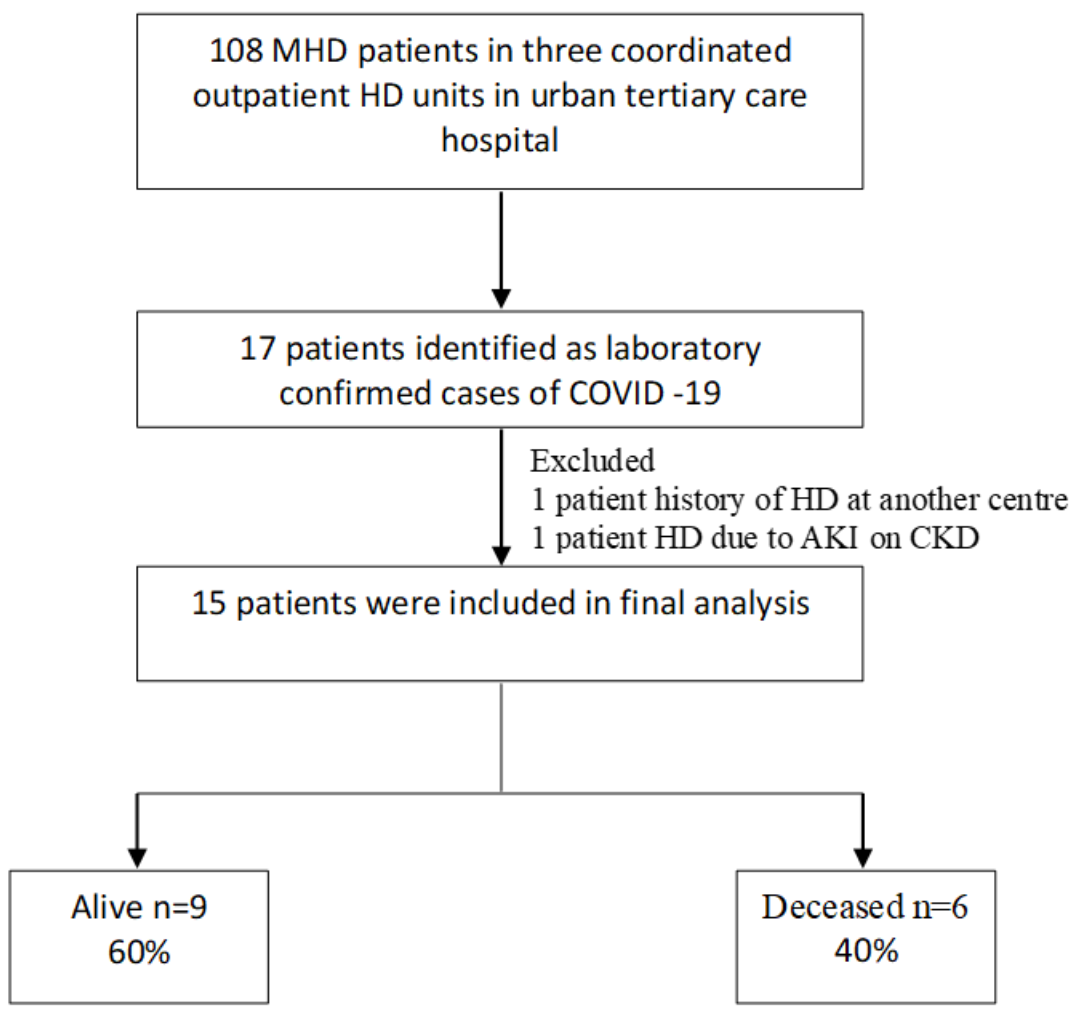

\section{Statistical Analysis:}

Continuous data was reported as mean \pm standard deviation $(\mathrm{SD})$ or median with interquartile range (IQR). Categorical variables were presented as frequency and percentage no. (\%). Outcome were compared using student $t$ test for normally distributed data and Man Whitney U test for skewed distribution. All p-values were based on two-sided tests and significance was set at a p-value less than 0.05. The analyses were performed using SPSS (Statistical Package of Social Sciences) version 19.

\section{Results:}

The incidence of COVID-19 observed in outpatient HD units at Aga Khan University Hospital, Karachi was $13.88 \%$. The mean age of study population was 62.8 years (SD \pm 8.6 ) and half of them were females. Most common cause of End Stage Renal Disease (ESRD) was diabetic kidney disease (80\%). All patients had at least one comorbid condition, hypertension being the most prevalent (100\%) followed by diabetes mellitus (86.7\%) and cardiovascular disease (33.3\%). One patient had active malignancy and was receiving chemotherapeutic treatment. None of the patients were on angiotensin converting enzyme inhibitors (ACEi) or angiotensin receptor blocker (ARB). All participants were receiving intermittent $\mathrm{HD}$ at the time of inclusion in this study. The median $\mathrm{HD}$ vintage was 15 months (interquartile range [IQR] 8-14). Vascular access in most patients was arteriovenous fistula (86.7\%) 
whereas $13.3 \%$ were being dialyzed through central venous catheter (CVC). Baseline characteristics of patients are presented in Table 1.

Figure 2: Detection of COVID-19 cases among 108 maintenance hemodialysis patients over time.

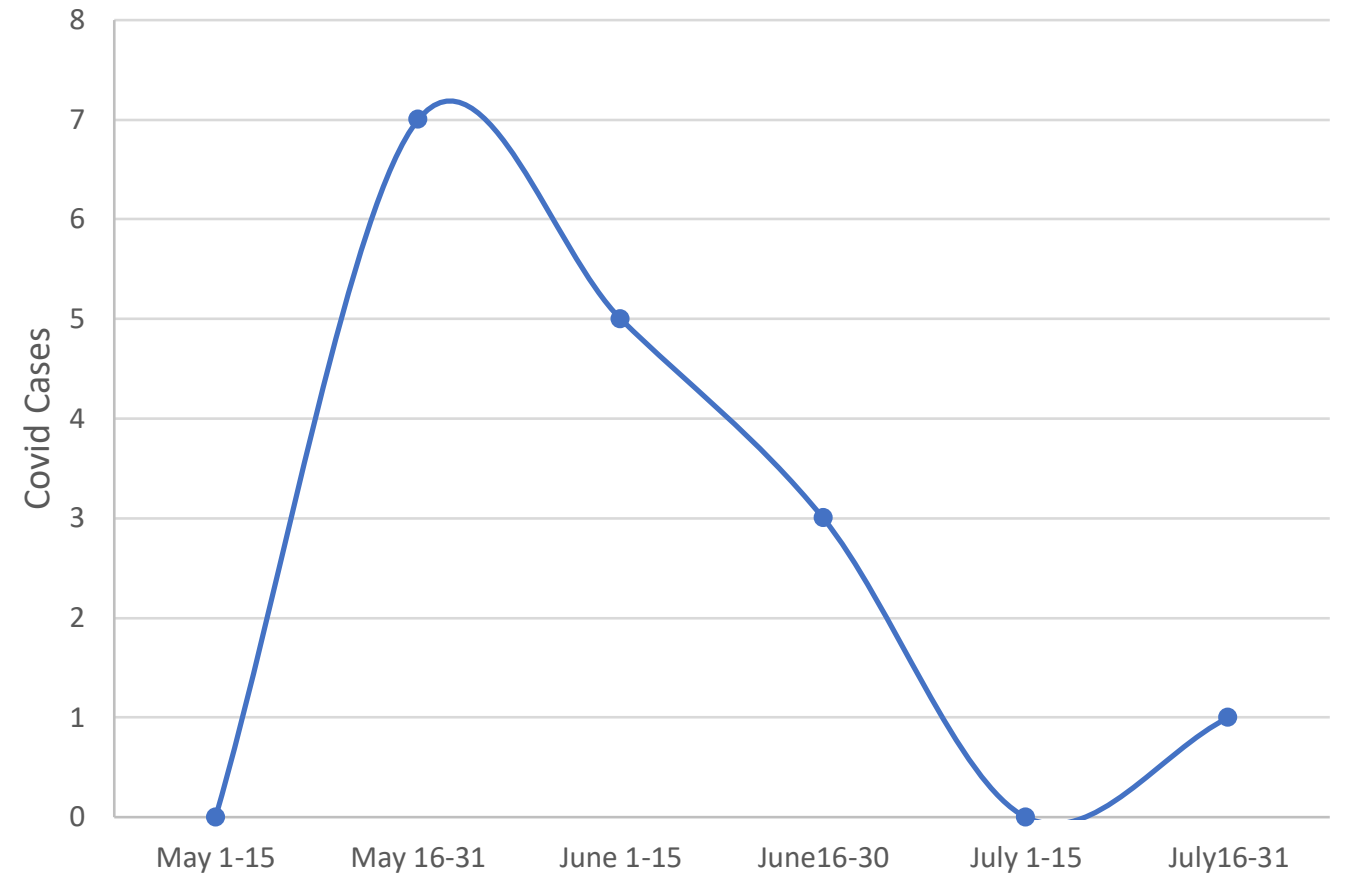

Forty percent of the patients were asymptomatic. Among those who had symptoms, most presented with shortness of breath (40\%) followed by cough (26.7\%), sputum production (20\%) and fever (20\%). Less common symptoms included fatigue, myalgia, sore throat, loss of appetite and loose stools. Initial chest radiographs showed infiltrates in $55.5 \%$ of patients either unilateral $(33.3 \%)$ or bilateral $(22.2 \%)$. The median hemoglobin level was 9.3 (IQR 7.9-10.7). Inflammatory markers were considerably raised. Median CRP, LDH, ferritin and PCT levels were 158.7(IQR 50.2-229.5), 472.5(IQR 244.7-667.5), 1305(IQR 1088-3718) and 2.04(IQR 1.5-39.4) respectively. Table 2 shows clinical, laboratory and radiologic findings.

Seven out of 15 patients (46.67\%) had at least one organ dysfunction. Median D-dimer value was 2.9 (IQR 1.1- 5.0). One patient (6.7\%) developed new thrombotic event in the form of clotted AVF and deep vein thrombosis during hospital stay. Three patients (20\%) developed acute respiratory distress syndrome (ARDS). Five patients (33.3\%) developed some degree of myocardial injury indicated by leaked troponin I while four patients (26.67\%) had hepatic dysfunction suggested by raised ALT and AST (37 [ IQR 23.7-43.2] and 74.5[IQR 51.2-89.7] respectively) as shown in Table 2 and 3.

Table 3 shows treatment and clinical outcomes in study population. Eight out of 15 (53.3\%) patients required hospitalization, however two refused hospital admissions and died. Five (33.3\%) of the hospitalized patients required ventilation either invasive (1 out of 5) or noninvasive (4 out of 5). Only one of them survived and 4 died including one who was intubated. Median length of hospital stay was 7.5 days (4-11.2). The mortality rate observed was $40 \%$ (6 out of 15). 
All patients continued to receive intermittent HD during course of illness. Seven of 15 (46.6\%) patients received broad spectrum antibiotics while none was given antivirals. Corticosteroid and vitamin-mineral supplements were given to six patients (40\%). One patient received convalescent plasma that showed no benefit.

Table 1: Baseline Characteristics of MHD Patients with COVID-19

\begin{tabular}{|c|c|c|c|c|}
\hline Characteristics & $\begin{array}{l}\text { All patients } \\
(n=15)\end{array}$ & Alive $(n=9)$ & Deceased $(n=6)$ & P-value \\
\hline $\begin{array}{l}\text { Age, years; mean } \pm S D \\
\text { Median (Range) }\end{array}$ & $\begin{array}{l}62.8 \pm 8.6 \\
62(57-66)\end{array}$ & $\begin{array}{l}59.7 \pm 8.9 ; 60(54- \\
62)\end{array}$ & $\begin{array}{l}67.3 \pm 6.4 ; \\
65.5(63.5-70.2)\end{array}$ & $0.01 *$ \\
\hline \multicolumn{4}{|l|}{ Gender } & \multirow{3}{*}{0.11} \\
\hline Male & $7(46.7)$ & $6(66.7)$ & $1(16.7)$ & \\
\hline Female & $8(53.3)$ & $3(33.3)$ & $5(83.3)$ & \\
\hline \multicolumn{4}{|l|}{ Causes of ESRD } & \multirow{6}{*}{0.28} \\
\hline DM & $12(80)$ & $6(66.7)$ & $6(100)$ & \\
\hline Unknown & $2(13.3)$ & $2(22.2)$ & 0 & \\
\hline Hypertension & 0 & 0 & 0 & \\
\hline Glomerulonephritis & 0 & 0 & 0 & \\
\hline Other & $1(6.7)$ & 1(11.1) & 0 & \\
\hline \multicolumn{4}{|l|}{ Comorbid } & \multirow[b]{2}{*}{ - } \\
\hline Hypertension & $15(100)$ & $9(100)$ & $6(100)$ & \\
\hline DM & $13(86.7)$ & $7(77.8)$ & $6(100)$ & 0.48 \\
\hline CVD & $5(33.3)$ & $3(33.3)$ & $2(33.3)$ & 0.99 \\
\hline COPD & $1(6.7)$ & $1(11.1)$ & 0 & - \\
\hline Malignancy & $1(6.7)$ & 0 & $1(16.7)$ & - \\
\hline \multicolumn{4}{|l|}{ Smoking status } & \multirow{3}{*}{0.89} \\
\hline Active & $2(13.3)$ & 1(11.1) & $1(16.7)$ & \\
\hline Ex-smoker & $2(13.3)$ & 1(11.1) & $1(16.7)$ & \\
\hline \multicolumn{4}{|l|}{ Exposure History } & \multirow{3}{*}{0.99} \\
\hline COVID-19 patient & $3(20.0)$ & $2(22.2)$ & $2(16.7)$ & \\
\hline $\begin{array}{l}\text { Family member with } \\
\text { COVID-19 }\end{array}$ & $1(6.7)$ & $1(11.1)$ & 0 & \\
\hline HD vintage (months) & $15(8-34)$ & $21.5(6.5-40)$ & $15(9-28)$ & 0.90 \\
\hline $\mathrm{KT} / \mathrm{V}$ & $1.19(1.06-1.37)$ & $1.19(1.07-1.4)$ & $1.13(0.98-1.28)$ & 0.28 \\
\hline \multicolumn{4}{|l|}{ Vascular access } & \multirow{3}{*}{0.99} \\
\hline CVC & $2(13.3)$ & 1(11.1) & $1(16.7)$ & \\
\hline AVF & $13(86.7)$ & $8(88.9)$ & $5(83.3)$ & \\
\hline \multicolumn{4}{|l|}{ Previous medication } & \multirow{4}{*}{$\begin{array}{l}- \\
- \\
-\end{array}$} \\
\hline ACEi & 0 & 0 & 0 & \\
\hline ARB & 0 & 0 & 0 & \\
\hline Immunosuppressant & $1(6.7)$ & 0 & $1(16.7)$ & \\
\hline
\end{tabular}

ESRD end stage renal disease; DM diabetes mellitus; CVD cardiovascular disease; COPD chronic obstructive pulmonary disease; HD hemodialysis; AVF arteriovenous fistula; CVC central venous catheter; ACEi angiotensin converting enzyme inhibitor; ARB angiotensin receptor blocker

Mean + SD; Median (IQR); n (\%) *Mann-Whitney U test

The comparison of clinical characteristics, laboratory and radiologic findings and outcomes between alive and deceased participants is shown in Tables 1-3. Patients who died were significantly older $(\mathrm{p}=$ 0.001). All deceased patients had diabetes mellitus as cause of renal failure, however no statistical significance was seen. There was no difference in comorbidities, history of smoking or exposure to COVID-19, HD vintage or access between the two groups. Symptoms at onset of illness were also similar in two groups. Shortness of breath was more common in deceased group. Better survival in asymptomatic patients was observed by the end of follow up period. However, this observation was not statistically significant. Inflammatory markers were higher in deceased group, more specifically CRP $(\mathrm{p}=0.06)$ and PCT $(\mathrm{p}=0.07)$. ARDS was a significant complication observed in those who died. 


\section{Discussion:}

The altered immune state together with associated comorbidities and impractical social distancing poses threat of severe COVID-19 in hemodialysis patients ${ }^{7,8}$. The risk may further increase in HD patients of low income countries due to lack of facilities and limitation of implementation of epidemiologic measures for infection control. ${ }^{17,}{ }^{18}$ Herein, we present the clinical characteristics and outcomes of COVID-19 in HD patients in Pakistan. To the best of our knowledge, this is the first study from South Asia to describe COVID-19 presentation and outcome in highly vulnerable HD population of this lowincome region.

Table 2: Clinical Presentation, Laboratory and Radiologic Findings in MHD patients with COVID-19

\begin{tabular}{|c|c|c|c|c|}
\hline Characteristics & All patients $(n=15)$ & Alive $(n=9)$ & Deceased $(n=6)$ & P-value \\
\hline \multicolumn{5}{|l|}{ Symptoms } \\
\hline Fever & $3(20)$ & $2(22.2)$ & $1(16.7)$ & 0.99 \\
\hline Fatigue & $2(13.3)$ & 1(11.1) & $1(16.7)$ & 0.99 \\
\hline Cough & $4(26.7)$ & $2(22.2)$ & $2(33.3)$ & 0.99 \\
\hline Sputum & $3(20)$ & 1(11.1) & $2(33.3)$ & 0.52 \\
\hline Shortness of breath & $6(40)$ & $2(22.2)$ & $4(66.7)$ & 0.52 \\
\hline Sore throat & $1(6.7)$ & 1(11.1) & 0 & - \\
\hline Diarrhea & $1(6.7)$ & 0 & $1(16.7)$ & - \\
\hline Anorexia & $1(6.7)$ & 0 & $1(16.7)$ & - \\
\hline Myalgia & $2(14.3)$ & 0 & $2(33.3)$ & - \\
\hline Asymptomatic & $6(40)$ & $4(44.4)$ & $2(33.3)$ & 0.99 \\
\hline \multicolumn{4}{|l|}{ CXR (initial) $n=9$} & \multirow{4}{*}{0.13} \\
\hline Clear lung fields & $4(44.4)$ & $1(25)$ & $3(60)$ & \\
\hline Unilateral infiltrates & $3(33.3)$ & $1(25)$ & $2(40)$ & \\
\hline Bilateral infiltrates & $2(22.2)$ & $2(50)$ & 0 & \\
\hline \multicolumn{5}{|l|}{ Laboratory parameters } \\
\hline $\mathrm{Hb} \mathrm{g/dL}$ & $9.3(7.9-10.7)$ & $8.8(7.8-10.3)$ & $9.7(8.8-12.1)$ & 0.32 \\
\hline TLC $10^{9} / \mathrm{L}$ & $9.4(6.9-10.5)$ & $8.2(6.2-9.8)$ & $10.4(6.2-21.9)$ & 0.19 \\
\hline Neutrophils $10^{9} / \mathrm{L}$ & $69.8(61-79.6)$ & $69.8(61-78.8)$ & 71.4(56.1-91.2) & 0.72 \\
\hline Lymphocytes $10^{9} / \mathrm{L}$ & $18(8.3-26)$ & $17.1(10.1-24.5)$ & $19.7(4.5-33.7)$ & 0.81 \\
\hline Platelets $10^{9} / \mathrm{L}$ & $241(179-319)$ & $209(163-264.5$ & $307(224.7-388.7)$ & 0.09 \\
\hline CRP $\mathrm{mg} / \mathrm{dL}(\mathrm{n}=12)$ & $158.7(50.2-229.5)$ & $50.7(5.7-191.5)$ & $213(167.5-304.4)$ & 0.06 \\
\hline LDH IU $/ L(n=8)$ & $472.5(244.7-667.5)$ & $323(319--)$ & $622(200-657)$ & 0.65 \\
\hline Procalcitonin $\mathrm{ng} / \mathrm{mL}(\mathrm{n}=7)$ & $2.04(1.5-39.4)$ & $1.5(0.37--)$ & $22.7(2.8-84.8)$ & 0.07 \\
\hline Ferritin $\mathrm{ng} / \mathrm{mL}(\mathrm{n}=11)$ & $1305(1088-3718)$ & $1521(999-3472)$ & $1157(769-5295)$ & 0.71 \\
\hline
\end{tabular}

Hb hemoglobin; TLC total leucocyte count; CRP C-reactive protein; LDH lactate dehydrogenase ${ }^{*} \mathrm{~N}(\%)$, Median (IQR)

The prevalence of COVID-19 observed in our HD units was $13.3 \%$ which is considerably higher compared to general population. ${ }^{19}$ Similar incidences were reported in many developed countries. Ninety-four out of 634 (15\%) HD patients acquired COVID-19 in a report from Brescia, Italy. ${ }^{13}$ Milia et al reported an incidence of $26 \%$ in their HD center in Lombardy, Italy. ${ }^{20}$ A study from Spain also reported similar incidence in HD population. ${ }^{12}$ Although experiences shared from Wuhan and France stated lower incidence of COVID-19 in ESRD, it was still higher than general population. ${ }^{7}{ }^{11}$ As discussed earlier, the inherent low immunity, comorbidity burden and failure to observe adequate social distancing in order to get life sustaining therapy may be the reason for higher incidence of COVID-19 in patients on HD. However, adopting recommended epidemiologic measures ${ }^{16}$, including vigilant screening and appropriate use of PPE may limit the spread of infection as observed in our study as well as in Chinese and Italian experiences. ${ }^{7,20}$ The impact of overall decline in COVID-19 cases in Pakistan on the reduction of infection rate observed in our center is however debatable. 
The prognosis of COVID-19 in HD patients is ambiguous and available data on mortality is wide-ranged depending on center and regional specificities. The case fatality rate in our study was $40 \%$ compared to $2.1 \%$ in general population. ${ }^{21}$ This was lower than $52 \%$ mortality reported in a large dialysis center in Italy ${ }^{20}$ and much higher than $14 \%$ seen in a Chinese cohort. ${ }^{22}$ Other single-center experiences from Europe and United States reported mortality rates ranging from $13 \%$ to $31 \%{ }^{8,9,11-13}$ This disparity could be due to differences in regional disease burden, healthcare resources and selection bias for hospitalization.

Table 3: Complications, Treatment and Short-term Outcome of COVID-19 in MHD patients

\begin{tabular}{|c|l|l|l|}
\hline Characteristics & All patients $(\mathrm{n}=\mathbf{1 5})$ & Alive $(\mathrm{n}=9)$ & Deceased ( $\mathrm{n}=6$ ) \\
\hline Complications & & & \\
\hline ARDS & $3(20)$ & 0 & $3(50)$ \\
\hline Myocardial Injury & $5(33.3)$ & $2(22.2)$ & $3(50)$ \\
\hline Hepatic Dysfunction & $4(26.7)$ & $2(22.2)$ & $2(33.3)$ \\
\hline Thrombotic Event & $1(6.7)$ & 0 & $1(16.7)$ \\
\hline Treatment & & & \\
\hline Antiviral & 0 & 0 & 0 \\
\hline Steroids & $6(40)$ & $4(66.7)$ & $2(33.3)$ \\
\hline Vitamin/mineral supplement & $6(40)$ & $2(22.2)$ & $4(66.6)$ \\
\hline Convalescent Plasma & $1(6.7)$ & 0 & $1(16.7)$ \\
\hline Need for Hospitalization & $6(40)$ & $2(22.2)$ & $4(66.7)$ \\
\hline Need for Ventilation & & & \\
\hline Noninvasive & $4(26.7)$ & $1(11.1)$ & $3(50)^{*}$ \\
\hline Invasive (mechanical) & $1(6.7)$ & 0 & $1(16.7)$ \\
\hline Length of Hospital Stay & $7.5(4-11.2)$ & $4(1-$ & $9(5.7-13.7)$ \\
\hline Cause of Death & & & \\
\hline ARDS & - & - & $2(33.3)$ \\
\hline Cardiac arrest & - & - & $2(33.3)$ \\
\hline Unknown & - & - & $2(33.3)$ \\
\hline
\end{tabular}

ARDS acute respiratory distress syndrome *advanced directive "Do Not Intubate"

Most of our patients were of older age than general population. Additionally, deceased patients were also significantly older than those still living $(\mathrm{p}=0.01)$. Similar observation was reported by Cunha et al in Spain ${ }^{12}$, Valeri et al in United States ${ }^{8}$ and Cécile et al in France ${ }^{11}$. Hypertension and diabetes were the most common comorbidities. They were also commonly found comorbidity in other studies ${ }^{7-9,13}$. Hypertension, diabetes, chronic kidney disease and age have been identified as risk factors for severe disease in general population ${ }^{2,3}$. High prevalence of these risk factors in dialysis patients could be the reason for high mortality rate observed in this group from COVID-19.

Unlike general population, fever was not a common symptom in our patients observed only in $20 \%$ of the cases. This was contrary to experience from Wuhan where 51.9\% HD patients had fever on presentation ${ }^{7}$. Similarly, Valeri et al reported fever in $49 \%$ of their HD patients in New York ${ }^{8}$. In a single-center study of hospitalized COVID-19 ESRD patients in Spain, 44\% patients had fever on admission and $89 \%$ developed during hospital stay ${ }^{12}$. The more prevalent symptom in our patients was shortness of breath seen in $40 \%$ and dry cough in $26.7 \%$ which were also common in Chinese, European and American population ${ }^{7-9,11,13,20,22}$. 


\section{COVID \& Maintenance Hemodialysis}

We found that a significant number of patients (40\%) were asymptomatic which is consistent with a report from a Spanish HD center where $40.5 \%$ of COVID-19 patients were asymptomatic ${ }^{10}$. Similarly, a report from China observed that $51 \%$ of 99 Covid- 19 patients from $5 \mathrm{HD}$ centers were asymptomatic at the time of diagnosis ${ }^{23}$. Six of 11 (55\%) SARS-CoV2 positive patients had no symptoms in HD unit in Canada ${ }^{24}$. The high prevalence of oligo-symptomatic and asymptomatic presentation in ESRD population poses challenge in prompt diagnosis of covid-19 in this group and emphasizes need for high clinical suspicion as well as implementation of proactive measures in screening and isolating suspected cases in order to mitigate an outbreak.

Unilateral or bilateral opacities and pulmonary edema on chest radiographic imaging is a common finding in COVID-19 ${ }^{25}$. Valeri et al reported 59\% bilateral opacities, $10 \%$ unilateral opacities and no radiographic findings in $9 \%$ of their study cohort ${ }^{8}$. Fisher and colleagues reported similar observation in $114 \mathrm{HD}$ patients with covid-19 in their study ${ }^{9}$. However, in our study we noted that $44.4 \%$ patients had no acute findings on initial chest x-rays. Likewise, in a single center experience from Spain, $56 \%$ patients had finding on chest $x$-ray whereas $44 \%$ had normal chest radiographs ${ }^{12}$. This is in line with Chinese observation where $40 \%$ of general population had no finding on chest x-ray, hence CT chest was universally performed for screening in China ${ }^{12}$.

Lymphopenia is a universal finding in covid-19 (29) and has also been observed in COVID-19 in HD patients $8,9,12,22,23$. Spontaneous lymphopenia can also occur in HD patients without underlying infection ${ }^{26}$, thus any inference should be drawn with caution. We did not find lymphopenia in initial work up, however development of lymphopenia later in the course of illness was not checked through serial monitoring of cell counts. In our study, inflammatory markers such as CRP, procalcitonin, LDH and ferritin were raised in all subjects. We also observed that compared to survivors, these markers were considerably higher in deceased group. Similar trend of inflammatory markers was also seen in Western cohorts $^{8,9,12}$. This is reflection of the fact that intensity of inflammatory response in covid-19 is directly associated with clinical outcome ${ }^{27,28}$. Hence serial monitoring of inflammatory markers may help in identifying severe cases facilitating timely intervention to improve disease outcome.

In our study, 53.3\% patients required hospitalization, $75 \%$ of whom got admitted in hospital and 25\% refused medical care and died at home. Among those who were admitted, $83.3 \%$ needed ventilation either invasive or noninvasive. Three of them (60\%) had given advanced directive of "Do Not Intubate" and died on noninvasive ventilation. The only patient who was intubated did not survive either. Since a significant number of patients opt out of medical intervention, we cannot ascertain the outcome of management had they received it. ARDS, myocardial injury, hepatic dysfunction and new thrombotic event were the complications observed in our study, where ARDS had worse outcome and all patients who developed ARDS died.

Strength of our study is that we comprehensively reviewed data of covid-19 in MHD patients for clinical presentation, laboratory and radiological findings, short term outcomes as well as impact of epidemiological intervention in a HD center of low income country. Furthermore, the dialysis units being situated in the largest metropolitan, our study population represent multiple ethnicities. This study also has some limitations. First, it is a quasi-single center study with small sample size and hence results cannot be generalized. Second, the data was collected retrospectively hence probability of incomplete documentation of medical information and missing investigations cannot be ruled out. Also, we included only PCR positive cases in this study and PCR-negative COVID-19 cases might have been overlooked. Moreover, due to small sample size it was not possible to determine risk factors for morbidity and mortality through multivariate adjusted analysis. Finally, given short follow up duration in this study, long-term impact of COVID-19 on morbidity and mortality could not be ascertained. For better 
understanding of COVID-19 in ESRD, large multicenter studies with more detailed patient information, timely investigation and longer follow up period are required.

\section{Conclusion:}

We therefore conclude that patients on MHD are highly vulnerable to severe COVID-19 and to adverse outcomes. Asymptomatic or oligo-symptomatic presentation can make diagnosis challenging for the nephrologists. High degree of clinical suspicion and vigilant screening together with implementation of strict preventive measures is required for effective control and management of this global pandemic. Inflammatory markers correlate with disease severity and mortality and following their trend may facilitate in appropriate management.

\section{Conflict of Interest: None}

\section{References:}

1. Robba C, Battaglini D, Pelosi P, Rocco PRM. Multiple organ dysfunction in SARS-CoV-2: MODS-CoV-2. Expert Rev Respir Med.1-4.

2. Garg S. Hospitalization rates and characteristics of patients hospitalized with laboratory-confirmed coronavirus disease 2019â $€$ ” COVID-NET, 14 States, March 1â€ “30, 2020. MMWR Morbidity and mortality weekly report.69.

3. Huang C, Wang Y, Li X, Ren L, Zhao J, Hu Y, et al. Clinical features of patients infected with 2019 novel coronavirus in Wuhan, China. Lancet.395(10223):497-506.

4. Saran R, Robinson B, Abbott KC, Agodoa LYC, Bhave N, Bragg-Gresham J, et al. US Renal Data System 2017 Annual Data Report: Epidemiology of Kidney Disease in the United States. Am J Kidney Dis.71(3 Suppl 1):A7.

5. Kato S, Chmielewski M, Honda H, Pecoits-Filho R, Matsuo S, Yuzawa Y, et al. Aspects of immune dysfunction in end-stage renal disease. Clin J Am Soc Nephrol. 2008;3(5):1526-33.

6. Stenvinkel P, Ketteler M, Johnson RJ, Lindholm B, Pecoits-Filho R, Riella M, et al. IL-10, IL-6, and TNF-alpha: central factors in the altered cytokine network of uremia--the good, the bad, and the ugly. Kidney Int. 2005;67(4):1216-33.

7. Xiong F, Tang H, Liu L, Tu C, Tian JB, Lei CT, et al. Clinical Characteristics of and Medical Interventions for COVID-19 in Hemodialysis Patients in Wuhan, China. J Am Soc Nephrol.31(7):1387-97.

8. Valeri AM, Robbins-Juarez SY, Stevens JS, Ahn W, Rao MK, Radhakrishnan J, et al. Presentation and Outcomes of Patients with ESKD and COVID-19. J Am Soc Nephrol.31(7):1409-15.

9. Fisher M, Yunes M, Mokrzycki MH, Golestaneh L, Alahiri E, Coco M. Chronic hemodialysis patients hospitalized with COVID-19-short-term outcomes in Bronx, New York. Kidney360.

10. Albalate M, Arribas P, Torres E, Cintra M, Alc Ãizar R, Puerta M, et al. High prevalence of asymptomatic COVID19 in hemodialysis. Daily learning during first month of COVID-19 pandemic. Nefrolog $\tilde{A}-\mathrm{a}$ (English Edition).40(3):279-86.

11. Cecile C, Florian B, Carole A, Clemence B, Philippe B, Francois C, et al. Low incidence of SARS-CoV-2, risk factors of mortality and the course of illness in the French national cohort of dialysis patients. Kidney Int.

12. Stock da Cunha T, Goma-Garces E, Avello A, Pereira-Garcia M, Mas-Fontao S, Ortiz A, et al. The Spectrum of Clinical and Serological Features of COVID-19 in Urban Hemodialysis Patients. J Clin Med.9(7).

13. Alberici F, Delbarba E, Manenti C, Econimo L, Valerio F, Pola A, et al. A report from the Brescia Renal COVID Task Force on the clinical characteristics and short-term outcome of hemodialysis patients with SARS-CoV-2 infection. Kidney Int.98(1):20-6.

14. Du X, Li H, Dong L, Li X, Tian M, Dong J. Clinical features of hemodialysis patients with COVID-19: a singlecenter retrospective study on 32 patients. Clinical and Experimental Nephrology.1.

15. Wang R, Liao C, He H, Hu C, Wei Z, Hong Z, et al. COVID-19 in Hemodialysis Patients: A Report of 5 Cases. Am J Kidney Dis.76(1):141-3.

16. CDC. Interim Additional Guidance for Infection Prevention and Control Recommendations for Patients with Suspected or Confirmed COVID-19 in Outpatient Hemodialysis Facilities [Internet]. 2020 [Available from: https://www.cdc.gov/coronavirus/2019-ncov/hcp/dialysis.html.

17. Ramachandran R, Jha V. Adding insult to injury: kidney replacement therapy during COVID-19 in India. Kidney Int.98(1):238-9.

18. Prasad N, Bhatt M, Agarwal SK, Kohli HS, Gopalakrishnan N, Fernando E, et al. The Adverse Effect of COVID Pandemic on the Care of Patients With Kidney Diseases in India. Kidney Int Rep.5(9):1545-50. 
19. Abid K, Bari YA, Younas M, Tahir Javaid S, Imran A. Progress of COVID-19 Epidemic in Pakistan. Asia Pac J Public Health.32(4):154-6.

20. La Milia V, Bacchini G, Bigi MC, Casartelli D, Cavalli A, Corti M, et al. COVID-19 Outbreak in a Large Hemodialysis Center in Lombardy, Italy. Kidney Int Rep.5(7):1095-9.

21. Tracker C. Pakistan COVID-19 Corona Tracker [Internet]. 2020 [Available from: https://www.coronatracker.com/country/pakistan/.

22. Wu J, Li J, Zhu G, Zhang Y, Bi Z, Yu Y, et al. Clinical Features of Maintenance Hemodialysis Patients with 2019 Novel Coronavirus-Infected Pneumonia in Wuhan, China. Clin J Am Soc Nephrol.15(8):1139-45.

23. Tang H, Tian JB, Dong JW, Tang XT, Yan ZY, Zhao YY, et al. Serologic Detection of SARS-CoV-2 Infections in Hemodialysis Centers: A Multicenter Retrospective Study in Wuhan, China. Am J Kidney Dis.76(4):490-9 e1.

24. Yau K, Muller MP, Lin M, Siddiqui N, Neskovic S, Shokar G, et al. COVID-19 Outbreak in an Urban Hemodialysis Unit. Am J Kidney Dis.

25. Wong HYF, Lam HYS, Fong AH, Leung ST, Chin TW, Lo CSY, et al. Frequency and Distribution of Chest Radiographic Findings in Patients Positive for COVID-19. Radiology.296(2):E72-E8.

26. FernÃindez-Fresnedo G, Ramos MaA, GonzÃilez-Pardo MC, de Francisco ALMn, LÃ $\tilde{p}^{3}$ ez-Hoyos M, Arias M. B lymphopenia in uraemia is related to an accelerated in vitro apoptosis and dysregulation of Bcl-2. Nephrology Dialysis Transplantation. 2000;15(4):502-10.

27. Zhang Y, Gao Y, Qiao L, Wang W, Chen D. Inflammatory Response Cells During Acute Respiratory Distress Syndrome in Patients With Coronavirus Disease 2019 (COVID-19). Ann Intern Med.173(5):402-4.

28. Ye Q, Wang B, Mao J. The pathogenesis and treatment of the 'Cytokine Storm' in COVID-19. J Infect.80(6):60713. 